\title{
Survival Analysis of Logistics Service Providers: An Empirical Study of Chengdu, Area in China
}

\author{
Guoqi Li1,2,3, Yihuan Tu${ }^{1}$, Lihao Duan ${ }^{1,2,3}$, Jin Zhang1,2,3, Lei Huang ${ }^{4}$, Wanshan Wu ${ }^{1,2}$, Zhuoshi Lv ${ }^{*}$ \\ ${ }^{1}$ School of Transportation and Logistics, Southwest Jiaotong University, Chengdu, Sichuan, China \\ ${ }^{2}$ National Engineering Laboratory of Integrated Transportation Big Data Application Technology, Chengdu, China \\ ${ }^{3}$ National United Engineering Laboratory of Integrated and Intelligent Transportation, Chengdu, China \\ ${ }^{4}$ School of Mathematics, Southwest Jiaotong University, Chengdu, China \\ Email: guoqi@swjtu.edu.cn, ${ }^{\star} 1103890664 @ q q . c o m$
}

How to cite this paper: Li, G.Q., Tu, Y.H., Duan, L.H., Zhang, J., Huang, L., Wu, W.S. and Lv, Z.S. (2020) Survival Analysis of Logistics Service Providers: An Empirical Study of Chengdu, Area in China. Open Journal of Statistics, 10, 915-935. https://doi.org/10.4236/ojs.2020.106054

Received: October 27, 2020

Accepted: November 29, 2020

Published: December 2, 2020

Copyright $\odot 2020$ by author(s) and Scientific Research Publishing Inc. This work is licensed under the Creative Commons Attribution International License (CC BY 4.0).

http://creativecommons.org/licenses/by/4.0/ (c) (i) Open Access

\begin{abstract}
This paper worked on a sample of 6791 logistics establishments registered in Chengdu, China over the period 1984-2016 to understand the survival status of logistics service providers (LSPs) by non-parametric Kaplan-Meier estimation, together with Cox proportional hazard regression model, to identify factors affecting the failure of LSPs. In particular, it studies the interaction effect between LSPs' size and entry timing and location. The empirical results show that: 1) Regarding the survival time, 1365 of the 6791 sample LSPs exited from the market by 2017 . The exit rate is $20.1 \%$, and the average life of the 6791 LSPs is about 6 years. 2) The survival of LSPs depends on their typology, ownership structure. And there is no significant difference in the probability of survival for both independent LSPs and logistics branches after controlling the effects of other variables. 3) Location and entry timing also play an important role in the survival of small-scale LSPs, but these factors cannot explain large-scale LSPs' failure.
\end{abstract}

\section{Keywords}

Logistics Service Providers, Survival Analysis, Kaplan-Meier Estimation, Cox Proportional Hazard Regression

\section{Introduction}

In China, the logistics industry is a basic and strategic industry and is widely valued by governments at all levels. Logistics is always playing an increasing role in the industrial structure transformation, transportation structure adjustment, supply chain innovations and domestic demand stimulus, and provides impor- 
tant support for high-quality economic development. As of 2019, the total cost of logistics in China reached 14.6 trillion yuan, which was 7.2 times that of 2001, from less than $30 \%$ of the United States in 2001 to 1.38 times of the United States. With the rapid increase in the size of the logistics market and the acceleration of the "metabolism" in the logistics industry, the survival problems of logistics enterprises have become increasingly prominent.

According to the "Analysis Report on the Survival Time of China Domestic Enterprises" by the China State Administration for Industry and Commerce [1], the average survival time of enterprises exited from transportation, warehousing, and postal services industry was 6.38 years over the period 2008 to 2012. Among these firms, the exit numbers were 311 thousand, accounting for $7.9 \%$ of the total firms that exited from the market in China, which was ranked fourth in all industries. Hence, it is urgent to analyze the factors influencing the survival of LSPs to help LSPs make correct business decisions and reduce the exit rate.

In this paper, we used a sample of 6791 LSPs to understand the post-entry performance of the logistics industry from 1985 to 2016 in Chengdu, China. As an important logistics hub city in the western region, the number of China-Europe freight trains via Chengdu has approached 1600 in 2018, ranking first in China for three consecutive years. In Chengdu, the logistics industry was considered as a strategic industry in 2004, and became an important factor in attracting foreign enterprises such as Intel and Foxconn. Therefore, it is of great practical significance and value to use Chengdu as a research area.

Existing research on the firm survival is mainly concentrated in the industrial field and rarely involves the service sector [2] [3], particularly the logistics industry. And a small number of studies focusing on the survival of service firms have also found significant differences in size, survival and growth with manufacturing enterprises [4] [5]. Hence, This paper draws upon the Resource-Based View of the firm, which contends firms are a heterogeneous bundle of tangible and intangible resources [6] [7], and applies the Cox proportional hazard regression model, combined with the Kaplan-Meier estimation, to identify the factors influencing the survival of LSPs. In addition, special emphasis is also placed on the interaction effect between LSPs' size and entry timing and location to clarify the impact mechanism efficiently.

The innovations and contributions of the paper are as follows: 1) The most important contribution of it is to indicate the relative importance of LSPs' type, ownership structure, location, entry timing, and size factor to the failure risk of LSPs. Especially through the interactive effects between LSPs' size and entry timing and location, it explains in detail the impact mechanism of LSPs' survival. 2) We try to apply new data sources to find research results that are different from existing manufacturing survival analysis. 3) It provides the evidence for the general statistics of logistics survival time in the existing logistics research. And our research method is also of universal significance in understanding the logistics survival problems of other cities in China, as well as Europe and the United 
States.

The rest of the paper is structured as follows. Section 2 reviews the relevant literature and proposes research hypothesis, while Section 3 is devoted to the data and variable description. Section 4 presents the picture of survival in the Chinese logistics industry by Kaplan-Meier estimation. Section 5 presents the empirical results. And the final part is the conclusions and managerial recommendations.

\section{Literature Review and Research Hypotheses}

The ultimate criterion for firm performance is the firm's survival [8]. And the length of survival is one of the most widely used measures of firm performance [9]. The earlier studies emphasize the entry and exit of the firms, focusing more on the impact of the firm's entry process, the exit risk and entry on market performance, and treated the survival process between the entry and exit as a "black box", and less on post-entry performance [10]. These researches have exaggerated the impact of entry on market performance [11]. Compared with the empirical data, what happens to firms subsequent to their entry is at least as important as the entry process itself [12]. As a result, the research on the post-entry performance has been increasing. A large number of relevant empirical studies on different manufacturing industries in different countries have been carried out [7] [13], becoming an important perspective to understand the industry dynamics and growth process.

The survival of firms depends on several factors, as summarized by Josef, et al. [14] and Manjón-Antolín and Arauzo-Carod [2], and these factors are mainly divided into two categories: internal factors and external factors. The internal factors refer to attributes, structural characteristics, and strategies of the new business itself, such as size, age, and ownership, etc. [15] [16] [17]. The external factors refer to the environment in which the firm operates, such as industry, geography, and economic cycle etc. [18] [19]. Based on the Resource-Based View of firm and firm survival literature, this paper studies the survival of LSPs from internal and external factors, and proposes the following hypotheses.

\subsection{Type of LSPs}

According to the Resource-Based View, resources are both heterogeneous distributed among firms and imperfectly mobile [6]. In the logistics industry, resources can be tangible (e.g. equipment, plants, fleets, hardware), or intangible (e.g. organizational processes, skills, know-how, reputation) [20]. Those heterogeneous resources across different LSPs may lead to different logistics performance and competitive advantage in the same market. And Lai [20] found that different types of LSPs do have significant differences in service performance. As service performance is related to the survival of firms, we hypothesized a link between the type of LSPs and the probability of survival.

H1. The likelihood of survival varies with the type of LSPs. 


\subsection{Branch}

According to Wang [21], logistics enterprises are composed of enterprise attribute elements and logistics attribute elements. In general, the enterprise attribute elements are usually including information center, command center, negotiation coordination, etc. which is common within the headquarters of logistics enterprises. While the logistics attribute elements are including equipment, warehouse, fleets, etc. tangible resources, which is more involved in logistics branches. Following this logic, the Resource-Based View suggests that distinct resources and capabilities between logistics headquarters and branches will lead to different levels of competitive performance. On the other hand, in literature on firm survival, there is also empirical evidence showing that non-branch entrants face lower exit risks than branch entrants [22] [23]. And many LSPs in this research sample have set up branches. This produced the following hypothesis:

H2. Non-branch LSPs have a better survival chance than their branch counterparts.

\subsection{Ownership}

There is empirical evidence showing that the ownership structure of firms matters in survival chances. Some authors have found that foreign-owned plants have a higher probability of exit than their domestic-owned counterparts in Ireland and Spanish manufacturing firms [24] [25] [26]. So, we proposed the following hypothesis:

H3. Foreign-owned LSPs have better survival prospects than their domestic-owned counterparts.

\subsection{Location}

Another dimension expected to affect the duration of firms, in the long run, is the location [23]. However, researches on the impact of location on the survival of firms have not yet reached a consistent conclusion. Fotopoulos and Louri [27] found that manufacturing companies located in Greater Athens have better survival prospects than others in the rest of the country, while Strotmann [22] found that manufacturing companies in rural areas have a lower risk of exit than their urban areas counterparts. However, in logistics literature, many studies have found logistics sprawl phenomenon, especially for new logistics establishments [28] [29]. This suggested a preference in location determine for LSPs. Based on those, we hypothesized a link between the location of LSPs and the probability of survival.

H4. LSPs in rural areas have better survival prospects than their urban area counterparts.

\subsection{Timing of Entry}

There is evidence that the timing of entry plays an important role in firm survival. According to Klepper [9] [30], enterprises that enter earlier are more likely to survive longer, because they can achieve higher profits in the early stages of the 
industry's life cycle. Hence, we proposed an entry timing hypothesis:

H5. Earlier entry LSPs have better survival prospects.

\subsection{Size}

The positive relationship between survival rate and size has been validated in numerous of empirical studies [31] [32]. Firms' size and age represent the efficiency differences arising from differences in experience, managerial abilities, production technology and firm organization. At the same time, larger firms have a lot of financial resources, and have advantages in raising social funds, obtaining tax incentives, and gathering high-quality human resources [7] [18] [33]. Therefore, we hypothesized a link between size and the probability of survival.

H6. The probability of survival positively depends on LSPs' size.

\section{Data Source and Variables}

\subsection{Data Source}

This paper utilizes a source of data set derived from the National Enterprise Credit Information Publicity System, an official database that includes information of all business registered establishment in china, which is constructed by the State Administration for Industry and Commerce. And it collects business registration information of data on industry and commercial establishments in China (e.g. enterprise name, registration date, date of dissolution, registered capital, address, business type, main business, etc.). The essential unit of observation in the system is the establishment, which is defined as a particular economic entity operating at a specific and single geographic location. Establishments in the logistics industry are frequently referred to LSPs. Compared with previous LSP-based literature, this paper used the system to track the changes of LSPs allows us to solve non-response bias problems in sample selection, which exists in many survey-based researches in the logistics industry [34].

We collected the data from the National Enterprise Credit Information Publicity System in December 2016 with the volume of 7012. After data cleaning, we created a representative sample of 6971 LSPs opened from 1984 to 2016 in Chengdu, of which 1365 LSPs closed in this period (20.1\%). Research on the survival problems of Chengdu's LSPs can provide an important perspective for understanding the survival conditions of Chinese LSPs, and it is also beneficial for government departments to develop strategies and policies for logistics industry development.

\subsection{Variable Description}

Our variable of interest is the duration of an LSP, defined as the time elapsed between the entry and the exit of the LSP, that is, the period between the foundation of the LSP (entry) and the end of its activities [5]. Information on entrants is based on the commercial registration date of the LSP. Likewise, information on exits is based on the dissolution date. For some LSPs, this period can 
be subject to right censoring (i.e. when the exit does not take place or still active). However, survival models can account for right censoring [35] [36]. Although the data is available up to December 2016, we prolonged the observation time to the next year (December 2017) in order to observe more exits of those opened in the last 3 years, since it is difficult to observe "deaths" for these young LSPs.

For H1, we worked with one categorical variable, Firm type, capturing attributes of the type of LSPs-transportation service providers (TSPs), warehousing service providers (WSPs), freight forwarding companies (FFCs) and integrated logistics service providers (ILSPs) are represented by 1, 2, 3 and 4 respectively. This classification is based on China's "Classification and Evaluation Index for Logistics Enterprise" and related scholars' research [20] [37].

For H2, we used one dummy variable, Branch, to measure whether an LSP is a branch or not-the variable that takes the value 1 when an LSP is a branch and 0 elsewhere.

For H3, we used one dummy variable, Ownership, to measure whether an LSPs belongs to foreign-owned or domestic-owned, the variable that takes the value 1 when an LSP is foreign-owned and 0 elsewhere. "Foreign-owned LSPs" in this research are defined as all types of foreign-funded LSPs, including LSPs from Hong Kong, Macao, and Taiwan, which provide logistics services for other manufacturing or commercial companies.

For H4, We worked with one dummy variable, Location, to capture different attributes of locations of LSPs, the variable that takes the value 1 when the firm is located at rural area and 0 elsewhere.

For H5, We worked with one categorical variable, Entry timing, to test for this hypothesis. The variable that takes the value 1 if the LSP's commercial registration date belongs to an early-stage (1984-2005), 2 if it is medium-stage (2006-2010), and 3 if it is late-stage (2011-2016). We choose 2006 and 2010 to divide the phase of the entry because the local municipal government in Chengdu began to implement the 5-year logistics plan in 2006. Another reason for choosing 2006 and 2010 as a split criterion is to allocate enough observations into each subgroup.

For H6, as we were interested in testing the effect of size, we used current registered capital (current refer to observation end date December 2017), to measure the size of the LSPs. Compared with initial size, the current size is found to be a better predictor of firm survival [31]. Then we followed Agarwal and Audretsch [38], distinguish between "small" and "large" firms by classifying firms as small if their current registered capital is less than the $60^{\text {th }}$ percentile of the registered capital distribution for all sample LSPs. Finally, we used one dummy variable, Size, to represent the size of LSPs. The variable that takes the value 1 for large scale and 0 for small scale.

Table 1 (the plots of the variable descriptive statistics are shown in Figure 1) shows the variable definitions and some descriptive statistics (e.g. the average LSPs duration was 72.488 months (about 6 years). 
Table 1. Description of variables.

\begin{tabular}{ll}
\hline Name of the Variable & Description \\
\hline Duration (months) & Survival time
\end{tabular}

Type of LSPs hypothesis

$\begin{array}{ll}\text { Firm type } & \text { categorical variable: } 1 \text { = transportation service providers (TSP); } \\ 2 \text { = warehousing service providers (WSPs); } 3=\text { freight forwarding } \\ \text { companies (FFCs); } 4 \text { = integrated logistics service providers (ILSPs) }\end{array}$

\section{Branch hypothesis}

Branch

\section{Ownership hypothesis}

Ownership

Location hypothesis

Location

\section{Timing of entry hypothesis}

Entry timing

Size

Dummy variable that takes the value 1 for large scale and 0 for small scale

Dummy variable that takes the value 1 if the LSP is branch and 0 elsewhere

Dummy variable that takes the value 1 if the LSP is foreign-owned and 0 elsewhere

Dummy variable that takes the value 1 if the LSP is located to rural area and 0 elsewhere

Categorical variable: 1,2 and 3 indicate the commercial registration date of LSPs in early-stage (1984-2005), medium-stage (2006-2010) and late-stage (2011-2016) respectively

\section{Size hypothesis}

scale

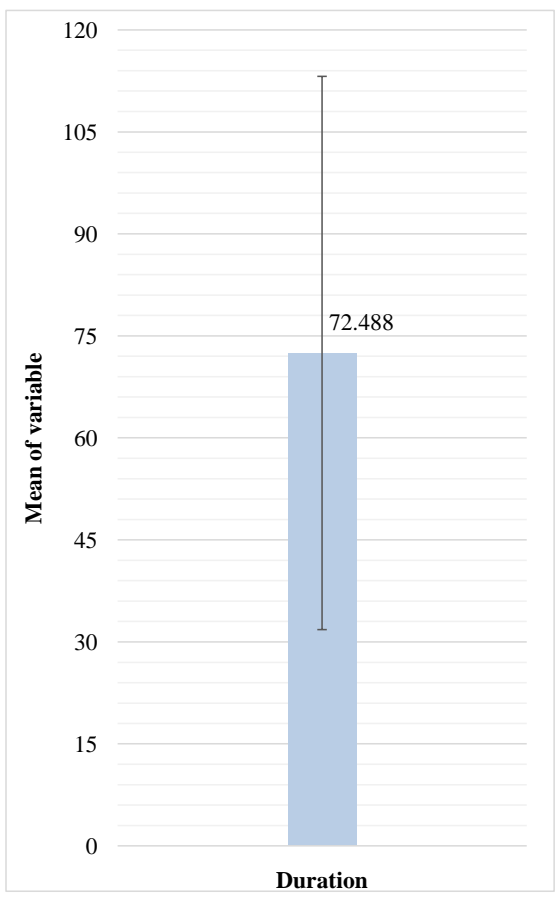

(a) 


\section{Kaplan-Meier Estimation of the Survival Time of the Samples}

The Kaplan-Meier estimator is the most widely used method for estimating survival functions, as it is a nonparametric maximum likelihood estimator with extremely few restrictions [10]. Indeed, the only restriction to consider is that the observed companies, if the data are censored, are assumed to have continued behaving the same way as they did until the death event occurred [5]. the survivor function $S(t)$ is the probability of survival past time $t$ or, equivalently, the probability of failing after $t$ [39]. The survival function is shown below:

$$
\hat{S}(t)=\prod_{j: t_{j} \leq t}\left(1-\frac{d_{j}}{n_{j}}\right)
$$

for $t_{1} \leq t \leq t_{k}$. Suppose there are $k$ distinct exit times, $t_{1} \leq t_{2} \leq \cdots \leq t_{k}$. At each time $t_{j}$, there are $n_{j}$ LSPs who are supposed to be at risk of an exit. Being at risk means they have not experienced an exit nor have they been censored prior to time $t_{j}$. If any cases are censored at exactly $t_{j}$, they are also considered to be at risk at $t_{j}$. Let $d_{j}$ be the number of LSPs who die on time $t_{j}$ [36].

Based on the Kaplan-Meier estimation method, this paper uses Stata14 to estimate the survival function of LSPs. The estimated survival curve by Kaplan-Meier is shown in Figure 2. In order to investigate whether the survival function of LSPs between $G$ groups corresponding to each variable are significantly different, we perform the log-rank test, which is the most widely used test for differences in the survival function [36]. Under the null hypothesis, the different groups of LSPs have the equivalent survival function. The test statistic is approximately chi-square in large samples with $G$-1 degrees of freedom, where $G$ denotes the number of groups corresponding to each covariable [40].

Table A1 (See Appendix) in the above shows the Kaplan-Meier survival estimates for different groups of LSPs. Considering the types of LSPs, ILSPs have the highest survival rate in terms of 5 years, 10 years and 15 years of survival. Its 15 -year survival rate is $92.4 \%$, which means that $92.4 \%$ of these LSPs survive for 15 years, nearly twice the TSPs and FFCs. In contrast, FFCs has the worst survival prospects, and its 5-year and 10-year survival rates are the lowest. Although the FFCs' 15 -year survival rate was $48.7 \%$ slightly higher than $48.5 \%$ of TSPs, the difference was not significant. The Kaplan-Meier survival estimates also show that the non-branch and foreign-owned LSPs have a higher survival rate than their branch and domestic-owned counterparts. In addition, LSPs located in rural area and those earlier entrants are more likely to survive. Furthermore, large scale LSPs are much more likely to survive than small scale ones, and their 15-year survival rate is nearly twice that of small-scale ones.

The results of the log-rank test show that the six variables reject the null hypothesis under the $1 \%$ significance, which indicates that there is a significant difference in survival functions of different groups of LSPs conditional on each covariate. For example, foreign-owned LSPs have much higher survival rates 
than their domestic-owned counterparts, and the difference of survival function between the two groups of LSPs is significant with P-value equal to 0.0011 . However, since Kaplan-Meier estimates do not control other explanatory variables that may affect LSPs' survival, this may result in that our estimates are not reliable. Therefore, in order to eliminate the estimation bias of the individual explanatory variable on the LSPs' survival, it is necessary to establish a multivariate analysis model-Cox proportional risk model to control other factors that affect the survival of LSPs.

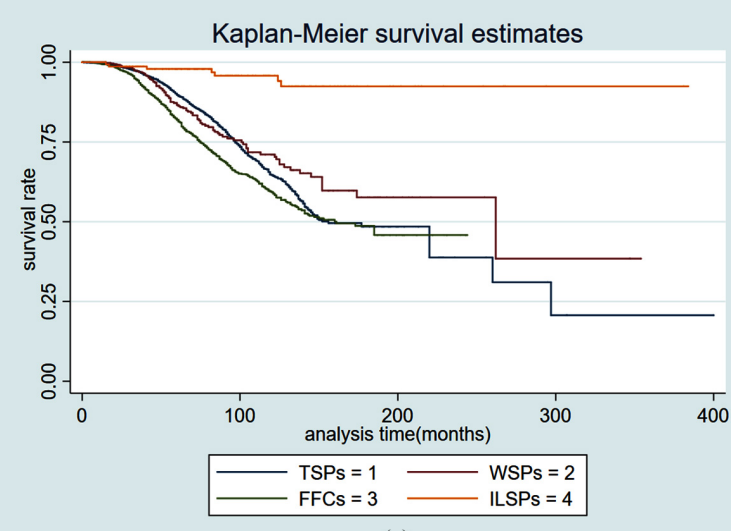

(a)

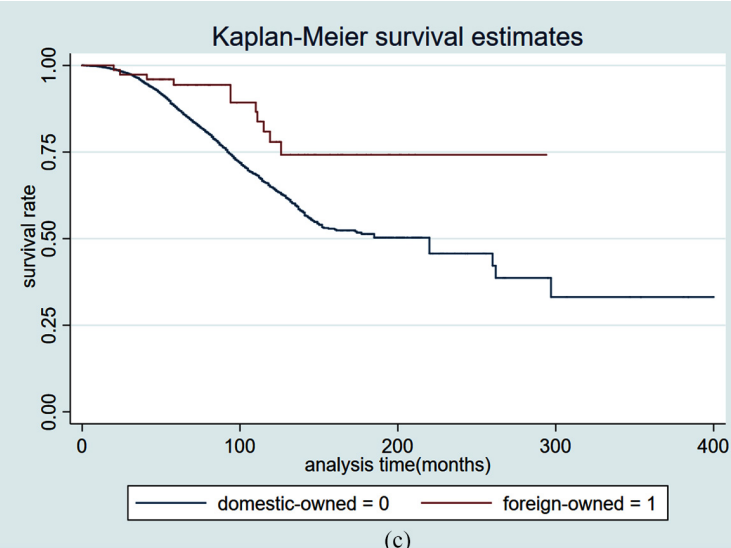

(c)

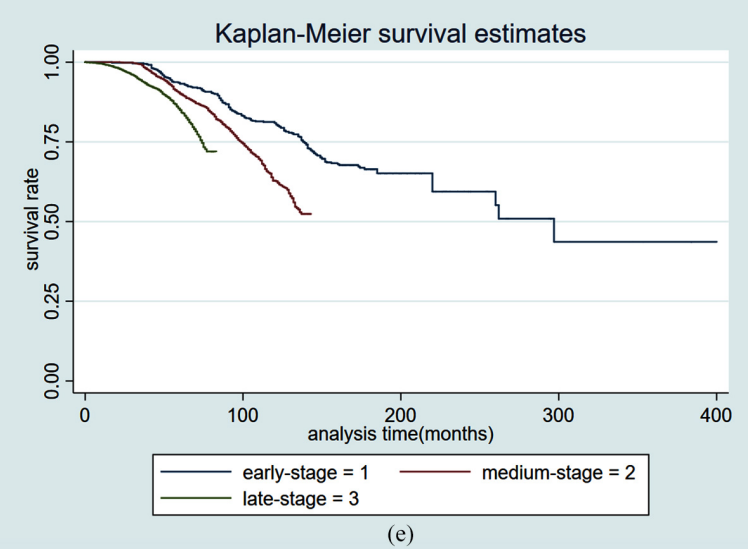

Figure 2. Plots of the Kaplan-Meier survival estimates with (a) Type of LSPs; (b) Branch; (c) Ownership; (d) Location; (e) Timing of entry; (f) Size.
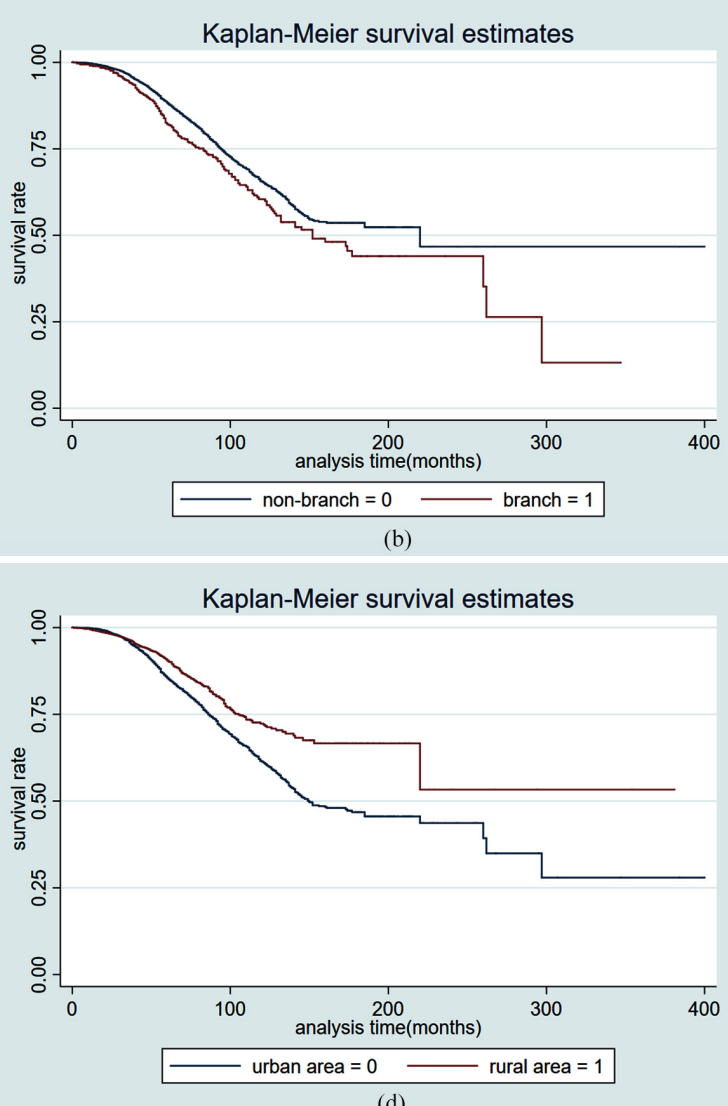

(d)

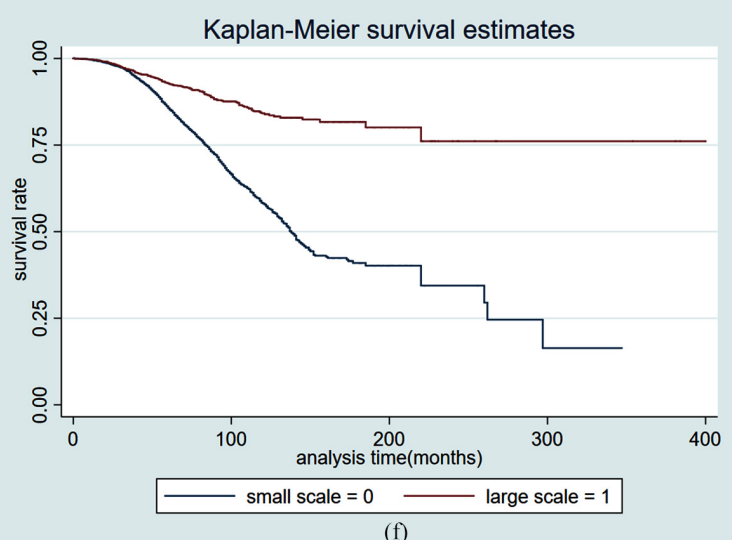




\section{Determinants of the Survival of LSPs}

\subsection{Model Design and Proportional Hazard Test}

In this section, we use the cox model [41] [42] to capture the effects of explanatory variables upon death (hazard rates) rather than upon times to death [43]. In addition, it corrects for the problem of censored data, which uses the following hazards model specification:

$$
h(t, X)=h_{0}(t) \exp \left(\beta_{1} x_{1}+\beta_{2} x_{2}+\cdots+\beta_{p} x_{p}\right)
$$

where $h(t, X)$ represents the hazard at time $t$ for an LSP with a given specification of a set of explanatory variables denoted by $X$. That is, the $X\left(x_{1}, x_{2}, \cdots, x_{p}\right)$ represents a vector of predictor variables that are being modeled to predict an LSP's hazard [40]. Where $\beta_{1}, \beta_{2}, \cdots, \beta_{p}$ is a set of unknown regression coefficients and $h_{0}(t)$ is an unknown non-negative baseline hazard function [43]. Through the partial maximum likelihood estimation, we estimate the value of $\beta_{1}, \beta_{2}, \cdots, \beta_{p}$, to determine the impact of these explanatory variables on the exit risk of LSPs.

The advantage of exponential specification is that the coefficient of one covariate (i.e. hazard ratio) can be directly explained as the constant proportional effect of a unit increase of this variable on the conditional probability of exiting. There are three assumptions in the proportional hazards model: 1) The LSPs do not influence the estimation of each model parameter. 2) Unobserved heterogeneity is assumed to be absent. 3) A suitable functional form exists for each assumed continuous covariate [5] [36].

Due to the fact that Firm type is a categorical variable, we introduce four dummy variables: TSPS, FFCs, WSPS and ILSPS (Dummy variable that takes the value 1 if an LSP belongs to that type and 0 otherwise), to evaluate the exit risk between different groups for each given covariate. To avoid multiple collinearities, we use ILSPS as a reference category. In the same way, we deal with the Entry timing variable, introducing three dummy variables: ES (early-stage), $M S$ (medium-stage) and LS (late-stage), dummy variable that takes value 1 if an LSP's commercial registration date belongs to corresponding period and 0 elsewhere. We also set $L S$ as the reference category. Therefore, the Cox proportional hazard model is initially set as follows:

$$
\begin{aligned}
h(t, X)= & h_{0}(t) \exp \left(\beta_{1} \text { TSPs }+\beta_{2} \text { FFCs }+\beta_{3} \text { WSPs }+\beta_{4}\right. \text { Branch } \\
& \left.+\beta_{5} \text { Ownership }+\beta_{6} \text { Location }+\beta_{7} E S+\beta_{8} M S+\beta_{9} \text { Size }\right)
\end{aligned}
$$

We use Stata14 to estimate the Cox proportional hazards model. The Cox likelihood is determined by the order of events (exits) and censorships and not by the distribution of the outcome variable [40]. In the partial maximum likelihood estimation of the Cox model, there are two important technical issues: one is the handling of the tied event, the other is to meet the assumption of proportional hazard (PH). If there are two or more exits of the LSP at the same time, known as "tied", we cannot identify which LSPs will exit firstly. As a result, it is imposs- 
ible to accurately identify the risk set of exit time [10]. And if the $\mathrm{PH}$ assumption cannot be satisfied, it is not appropriate to apply the Cox proportional hazard model. To achieve the goal, we use the exact method to deal with tied events, which considers all possible sequences of exits at each point in time of tie, obtaining more accurate parameter estimation results [36]. To test the $\mathrm{PH}$ assumption, we first used the Schoenfeld residual method, which is especially suitable for large samples, to verify whether the model meets the applicable conditions.

Schoenfeld residual method's idea is to retrieve the residuals, fit a smooth function of time to them, and then test whether there is a relationship. Because the variables selected in this paper doesn't vary with time, we use its simplest form. When there are no tied failure times, the Schoenfeld residual for covariate $x_{u}, u=1, \cdots, p$, and for observation $j$ observed to fail is

$$
r_{u j}=x_{u j}-\frac{\sum_{i \in R_{j}} x_{u i} \exp \left(X_{i} \hat{\beta}_{x}\right)}{\sum_{i \in R_{j}} \exp \left(X_{i} \hat{\beta}_{x}\right)}
$$

That is, $r_{u j}$ is the difference between the covariate value for the failed observation and the weighted average of the covariate values (weighted according to the estimated relative hazard from a Cox model) over all those subjects at risk of failure when subject $j$ failed [39].

The test results are shown in Table A2 (See Appendix). Based on Grambsch and Therneau [44], the null hypothesis that the hazard rates are proportional over time for Size variable is violated at the five percent level, with the p-value is 0.000 (The $\mathrm{P}$ values of other variables were all higher than 0.05 ). Therefore, it is necessary to adjust for the Model (3).

\subsection{Model Adjustment and Analysis Results}

Since the PH assumption is not satisfied in the Model (3), we choose the extended Cox model. Through implementing hierarchical control of variables that do not meet the $\mathrm{PH}$ assumption, so that variables that do not satisfy $\mathrm{PH}$ assumption are excluded in the model. Furthermore, considering the possible interaction effect between LSPs' size and entry timing and location, we introduce three product terms: Size $\times$ Location, Size $\times E S$, and Size $\times M S$, which are represented by $S \_L, S \_E S$, and $S \_M S$ respectively. Therefore, the Cox hazard model with product terms stratified by Size variable is as follows:

$$
\begin{aligned}
h_{g}(t, X)= & h_{0_{g}}(t) \exp \left(\beta_{1} \text { TSPs }+\beta_{2} \text { FFCs }+\beta_{3} \text { WSP }+\beta_{4}\right. \text { Branch } \\
& +\beta_{5} \text { Ownership }+\beta_{6} \text { Location }+\beta_{7} E S+\beta_{8} M S \\
& \left.+\beta_{9} S \_L+\beta_{10} S \_E S+\beta_{11} S \_M S\right) .
\end{aligned}
$$

where $g=0$ means Size $=0$, and $g=1$ means Size $=1$. Using Stata 14, we choose the method of the exact marginal likelihood to handle the tied event. The estimated results of the Model (4) are shown in Figure 3, and the detailed results are given in Table A3 (See Appendix). 


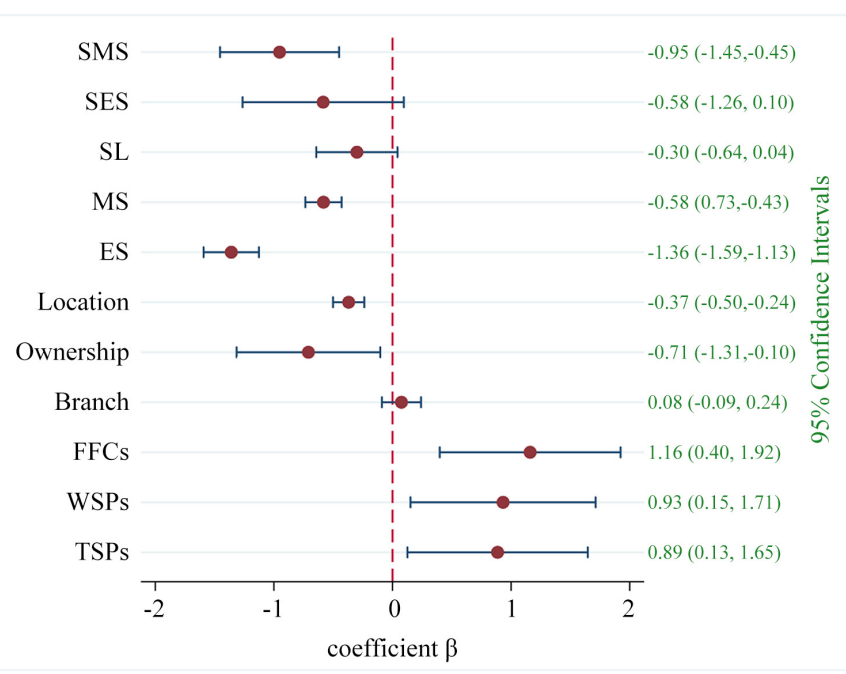

Figure 3. Stratified Cox proportional hazard model of Model 4 (coefficient $\beta$ ).

The Hazard Ratio in Table A3 (See Appendix) indicates the effect on the risk of exiting, can be interpreted almost exactly like odds ratios in logistic regression (i.e. for dummy variables with values of 1 and 0 , it can be interpreted as the ratio of the estimated hazard for those LSPs with a value of 1 to the estimated hazard for those with a value of 0 after controlling for other variables) [36]. The results of Models (1), (2), (3), (4) show:

Hypothesis 1 concerns on the impact of the type of LSPs on survival. After control for other variables and at the 5\% level of significance, the four types of LSPs face the risk of exit is ISLPs $<$ TSPs $<$ WSPs $<$ FFCs. The hazard ratio for the FFCs are 3.1919, implying that the odds of the FFCs of exiting the market are more than three times that of the ISLPs, controlling for all other variables. A possible explanation is that, unlike the other three types of LSPs, ISLPs have differentiated competitive advantages. In addition to providing basic transportation, warehousing, and freight forwarding services, they also provide more diversified service offerings, such as logistics system design and information management. Finally, the results support that the performance of integrated service logistics providers is the best while freight forwarders are worst [20]. Hence, Hypothesis 1 is accepted.

Hypothesis 2 pays attention to the difference between the survival of branch and non-branch LSPs. Although Kaplan-Meier estimation indicates that the branch group has a shorter survival time than their non-branch group, there is no significant difference in the survival experience of the two groups after controlling the effects of other variables. From the perspective of the supply chain, the branch can be regarded as a partner in the outsourcing of an independent LSP. That is to say, the branch is a link in the logistics process, and its operating results are directly related to the performance of the entire supply chain members. In addition, Ono [45] found that due to the dependence of the branch on headquarters or other affiliates, the branch has a lower dependence on external suppliers and has less operational risk than independent enterprises. Therefore, 
the survival status of the branch is not necessarily worse than that of the independent LSPs. This is consistent with Audretsch and Mahmood [43] who found that the difference in survival experience between the branch and the non-branch is caused by the characteristics of the firm and the external environment, rather than its own reasons. Hence, hypothesis 2 is rejected.

Hypothesis 3 focuses on the impact of the ownership structure of the LSPs on survival. At the $5 \%$ level of significance, the exit risk of foreign-owned LSPs is nearly half of domestic-owned, after controlling for other variables, which shows that foreign-owned LSPs have better survival prospects, which echoes with the findings of Hull, et al. [46]. The possible explanation is that the foreign-owned LSPs entering the Chinese market are large-scale and have strong anti-risk capabilities. Secondly, foreign enterprises have rich management experience and stable customers. Therefore, the survival and development of foreign-funded enterprises are better.

Considering the interactions effect of location, entry timing and size in hypotheses 4, 5 and 6, we find that the Model (4) had the best fitting effect, and its corresponding p-value was 0.0004 in 3 degrees of freedom through the likelihood ratio test. Therefore, we use the results of the Model (4) to verify these three assumptions.

Hypothesis 4 concerns on the impact of location on survival. After controlling other variables, the exit risk of LSPs located in the rural area is about $\exp \left(\beta_{6} \times 1+\beta_{9} \times\right.$ Size $\left.\times 1\right)$ times that of urban areas. The results are divided into two categories: 1 ) When Size $=1$ (large-scale LSPs), at the $10 \%$ level of significance, the exit risk in rural area is 0.5119 times that of the urban area; 2) When Size $=0$ (small-scale LSPs), at the $1 \%$ level of significance, the exit risk in the rural area is 0.6910 times that of the urban area. This shows that the exit risk of LSPs located in the suburbs is lower than that of urban LSPs, especially for small-scale ones ( $1 \%$ significance level). A possible explanation is that due the high land price and increased congestion, land shortage, and lack of logistics infrastructures, such as parking and loading space, the urban core area is no longer suitable for organizing logistics services [47]. Furthermore, social conditions in the suburbs are more attractive to LSPs, such as lower staff wages and higher acceptance of tough logistics jobs due to lack of job opportunities and higher unemployment [29]. Finally, many suburban local governments have adopted favorable land-use policies, environmental standards, and financial incentives to attract logistics investment, which also contributes to the survival of LSPs to some degree. However, for large-scale LSPs, the impact of location on their survival remains to be further tested, since this conclusion is only established at a level of significance of $10 \%$. One possible interpretation is that large enterprises are usually more self-sufficient and therefore less dependent on external resources. In addition, small-scale LSPs are more sensitive to labor costs than large ones [48].

Hypothesis 5 focuses on the impact of the entry timing on survival. After con- 
trolling other variables, there are two stages: 1$)$ The exit risk of the LSPs registered in 1984-2005 is $\exp \left(\beta_{7} \times 1+\beta_{10} \times\right.$ Size $\left.\times 1\right)$ times that of registered in 2011-2016. The results are subdivided into two types: 1-1 When Size $=0$, at the $1 \%$ level of significance, the exit risk of the LSPs registered in 1984-2005 is 0.2568 times that of registered in 2011-2016; 1-2 When Size $=1$, at the $10 \%$ level of significance, the exit risk of the LSPs registered in 1984-2005 is 0.1431 times that of registered in 2011-2016. 2) The exit risk of the LSPs registered in 2006-2010 is the $\exp \left(\beta_{8} \times 1+\beta_{11} \times\right.$ Size $\left.\times 1\right)$ times of registered in 2011-2016. The results are subdivided into two types: $2-1$ When Size $=0$, at the $1 \%$ level of significance, the exit risk of the LSPs registered in 2006-2010 is 0.5588 times that of registered in 2011-2016; 2-2 When Size =1, at the 1\% level of significance, the exit risk of LSPs registered in 2006-2010 is 0.2156 times that of registered in 2011-2016.

At the 5\% level of significance, we can only confirm that for small-scale LSPs, the exit risk of LSPs registered in 1984-2005 is 0.2568 times that of registered in 2011-2016. The exit risk of LSPs registered in 2006-2010 is 0.5588 times that of registered in 2011-2016. The earlier the registration date, the lower the exit risk of LSPs. The possible explanation is that the earlier entrants face a higher industry price-cost margin and thus conducts more $R \& D$ and grows more than the later entrants. However, for large-scale LSPs, the same finding just only valid for mid-to-late entrants, not include early entrants. The possible explanation is that market forces in the early stage were mainly regulated by the government. With China's accession to the World Trade Organization (WTO) in 2001, the Chinese logistics market began to gradually open to foreign investment [49]. Since then, the market mechanism has begun to play a greater role in the Chinese logistics industry. Therefore, economies of scale are beginning to work.

Hypothesis 6 focuses on the impact of size on survival. Because the size variable is a hierarchical variable that is not directly incorporated into the model. Therefore, it is impossible to directly compare the exit risks of large-scale and small-scale LSPs. We can only find that large scale LSPs are much more likely to survive than small scale ones in Kaplan-Meier estimation without controlling other variables. Hence, the impact of size on LSPs' survival remains to be further confirmed.

\section{Conclusions and Recommendations}

The exit of firms in multiple fields, such as mining, manufacturing, medicine and technology industry, has been widely investigated. However, it seems to be the first study to analyze the exit of firms in logistics industry. Little is consequently known about what determines firm exit in logistics industry. Therefore, this paper investigates the factors influencing the survival of LSPs in Chengdu, China over the period 1984-2016. Over this thirty-three-year period, roughly 20 percent of 6791 LSPs exited from the market, and the average life is about 6 years. In order to investigate the determinants of survival of LSPs, we used the 
Stratified Cox proportional hazard model. In particular, an important difference with previous researches on firm survival is that our model incorporated interaction effect between LSPs' size and entry timing and location. And there are indeed findings that are different from the survival of manufacturing enterprises. Conclusions as below:

1) The survival of LSPs depends on their typology, ownership structure. There is no significant difference in the probability of survival for both independent LSPs and logistics branches after controlling the effects of other variables.

2) There is no significant difference in survival probability between earlier and later entrants of large-scale logistics enterprises. This is different from Klepper [9], who did not consider interaction effect between firm size and entry timing had found the earlier manufacturers entered the US automobiles, tire, television, and penicillin industries, the lower hazards they faced. The possible reason is that the earlier entrants with large-scale in logistics were mostly state-owned enterprises, which underwent a change of ownership during the reform and opening-up process and became new enterprises.

3) There is also a difference regarding the effect of location on firm survival. For example, for small-scale LSPs, rural area LSPs have lower hazards than the corresponding risk in urban areas, while this trend cannot be accepted at 5\% significance for large-scale LSPs. This finding is different from Strotmann [22], who found that manufacturing companies in rural areas have a lower risk of exit than their urban areas counterparts. This is closely related to the trend of suburbanization of Chinese logistics enterprises [28] [50]. Due to the lack of fixed resources, such as high-standard warehouses and railway dedicated lines, small LSPs are the main objects to be relocated under the pressure of environment and congestion in central urban areas. In addition, the Chinese government has established many public logistics parks in the suburbs and has provided many supporting policies for enterprises moving into the park. This has driven small logistics enterprises to continuously relocate to suburban logistics parks and achieve better survival.

Based on the statistical research of survival of LSPs, this paper puts forward the following recommendations:

1) The survival prospects of integrated logistics enterprises are the best, which shows that diversified operations are conducive to reducing operating risks. However, the excessive degree of diversification will distract the attention of LSPs and have a negative impact on survival. Therefore, LSPs need to pay attention to the degree of diversification.

2) The empirical results in this paper have confirmed the importance of ownership structure on LSPs' survival. Hence, the government should lower the barriers to entry for foreign-owned logistics enterprises so that they can enter a wider market, thereby promoting comprehensive and healthy competition between foreign-owned and domestic-owned logistics enterprises. Meanwhile, local logistics enterprises should strengthen strategic cooperation and knowledge 
sharing with foreign companies, improving their professional service capabilities, and especially improve their competitiveness by providing services to foreign manufacturing enterprises.

3) For small-scale LSPs, location and entry timing have a significant impact on their survival. Being located in the suburbs is conducive to the survival of small LSPs illustrates the importance of location for small logistics enterprises to acquire and employ resources. Because lower land prices in the suburbs can reduce LSPs operating costs and the environmental impact of logistics activities in the central urban area, improving land utilization of logistics land and reducing the multiple transfers of land is of great significance to the survival of logistics enterprises. Meanwhile, the later a small LSP enters the market, the higher the exit risk. This shows that the intensified market competition has had an important impact on the survival of small logistics enterprises. Decision-makers should encourage the merge and alliance of small logistics enterprises in some industries with excessive competition, such as road transportation, warehousing, and freight forwarding, to jointly improve their ability to resist risks.

\section{Fund Project}

National Natural Science Foundation of China [71603219, 41501123], Applied Basic Research Project of Science and Technology Office of Sichuan Province [2019YJ2017].

\section{Conflicts of Interest}

The authors declare no conflicts of interest regarding the publication of this paper.

\section{References}

[1] China State Administration for Industry and Commerce (2013) Analysis Report on the Survival Time of Domestic Enterprises in China. China Development Observation, 28-32.

[2] Manjón-Antolín, M.C. and Arauzo-Carod, J.-M. (2008) Firm Survival: Methods and Evidence. Empirica, 35, 1-24. https://doi.org/10.1007/s10663-007-9048-x

[3] Coad, A. and Guenther, C. (2012) Diversification Patterns and Survival as Firms Mature. Small Business Economics, 41, 633-649. https://doi.org/10.1007/s11187-012-9447-7

[4] Audretsch, D.B., Klomp, L. and Thurik, A.R.R. (1998) Do Services Differ from Manufacturing? The Post-Entry Performance of Firms in Dutch Services. Social Science Electronic Publishing, Rochester.

[5] Gémar, G., Moniche, L. and Morales, A.J. (2016) Survival Analysis of the Spanish Hotel Industry. Tourism Management, 54, 428-438. https://doi.org/10.1016/j.tourman.2015.12.012

[6] Barney, J. (1991) Firm Resources and Sustained Competitive Advantage. Journal of Management, 17, 99-120. https://doi.org/10.1177/014920639101700108

[7] Esteve-Pérez, S. and Mañez-Castillejo, J.A. (2008) The Resource-Based Theory of the Firm and Firm Survival. Small Business Economics, 30, 231-249. 
https://doi.org/10.1007/s11187-006-9011-4

[8] Reimann, B.C. (1982) Organizational Competence as a Predictor of Long Run Survival and Growth. The Academy of Management Journal, 25, 323-334. https://doi.org/10.2307/255994

[9] Klepper, S. (2002) Firm Survival and the Evolution of Oligopoly. The RAND Journal of Economics, 33, 37-61. https://doi.org/10.2307/2696374

[10] Xiao, X., He, W. and Guo, X. (2014) Capacity Accumulation, Expansion Behavior and the Duration of Enterprise Survival: A Study of Enterprise Survival in China's Strategic Emerging Industries. Management World, 2, 77-89.

[11] Geroski, P. and Schwalbach, J. (1991) Entry and Market Contestability: An International Comparison. Blackwell, Oxford.

[12] Audretsch, D.B. and Mata, J. (1995) The Post-Entry Performance of Firms: Introduction. International Journal of Industrial Organization, 13, 413-419.

https://doi.org/10.1016/0167-7187(95)00497-1

[13] Ebert, T., Brenner, T. and Brixy, U. (2018) New Firm Survival: The Interdependence between Regional Externalities and Innovativeness. Small Business Economics, 53, 287-309. https://doi.org/10.1007/s11187-018-0026-4

[14] Josef, B., Peter, P. and Ziegler, R. (1992) Survival Chances of Newly Founded Business Organizations. American Sociological Review, 57, 227-242.

https://doi.org/10.2307/2096207

[15] Geurts, K. and Van Biesebroeck, J. (2016) Firm Creation and Post-Entry Dynamics of de Novo Entrants. International Journal of Industrial Organization, 49, 59-104. https://doi.org/10.1016/j.ijindorg.2016.08.002

[16] Zhang, D., Zheng, W. and Ning, L. (2018) Does Innovation Facilitate Firm Survival? Evidence from Chinese High-Tech Firms. Economic Modelling, 75, 458-468. https://doi.org/10.1016/j.econmod.2018.07.030

[17] Chlebicka, A. and Pietrzak, M. (2018) Size of Membership and Survival Patterns of Producers' Organizations in Agriculture-Social Aspects Based on Evidence from Poland. Sustainability, 10, 2293. https://doi.org/10.3390/su10072293

[18] Geroski, P.A., Mata, J. and Portugal, P. (2010) Founding Conditions and the Survival of New Firms. Strategic Management Journal, 31, 510-529. https://doi.org/10.1002/smj.823

[19] Arcuri, G., Brunetto, M. and Levratto, N. (2018) Spatial Patterns and Determinants of Firm Exit: An Empirical Analysis on France. The Annals of Regional Science, 62, 99-118. https://doi.org/10.1007/s00168-018-0887-0

[20] Lai, K. (2004) Service Capability and Performance of Logistics Service Providers. Transportation Research Part E: Logistics and Transportation Review, 40, 385-399. https://doi.org/10.1016/j.tre.2004.01.002

[21] Wang, C. (2008) Spatial Organizational Network of Logistics Company in China. Acta Geographica Sinica, 63, 135-146.

[22] Strotmann, H. (2006) Entrepreneurial Survival. Small Business Economics, 28, 87-104. https://doi.org/10.1007/s11187-005-8859-z

[23] Wang, C. and Giouvris, E. (2020) Firm Survival between Manufacturing and NonManufacturing Industries: Cultural Distance, Country Risk, Entry Mode, Market Size, Firm Age and Location. The Chinese Economy, 53, 412-431. https://doi.org/10.1080/10971475.2020.1730564

[24] Pérez, S.E., Llopis, A.S. and Llopis, J.A.S. (2004) The Determinants of Survival of Spanish Manufacturing Firms. Review of Industrial Organization, 25, 251-273. 
https://doi.org/10.1007/s11151-004-1972-3

[25] Baumöhl, E., Iwasaki, I. and Kočenda, E. (2020) Firm Survival in New EU Member States. Economic Systems, 44, Article ID: 100743.

https://doi.org/10.1016/j.ecosys.2020.100743

[26] Iwasaki, I. and Kim, B.-Y. (2020) Legal Forms, Organizational Architecture, and Firm Failure: A Large Survival Analysis of Russian Corporations. European Journal of Law and Economics, 49, 227-275. https://doi.org/10.1007/s10657-020-09644-8

[27] Fotopoulos, G. and Louri, H. (2000) Location and Survival of New Entry. Small Business Economics, 14, 311-321. https://doi.org/10.1023/A:1008180522759

[28] Liu, S., Li, G. and Jin, F. (2018) Quantitative Measurement and Development Evaluation of Logistics Clusters in China. Journal of Geographical Sciences, 73, 1540-1555.

[29] Strale, M. (2019) Logistics Sprawl in the Brussels Metropolitan Area: Toward a Socio-Geographic Typology. Journal of Transport Geography, 88, Article ID: 102372. https://doi.org/10.1016/j.jtrangeo.2018.12.009

[30] Klepper, S. (1996) Entry, Exit, Growth, and Innovation over the Product Life Cycle. The American Economic Review, 86, 562-583.

[31] Mata, J., Portugal, P. and Guimarães, P. (1995) The Survival of New Plants: Start-Up Conditions and Post-Entry Evolution. International Journal of Industrial Organization, 13, 459-481. https://doi.org/10.1016/0167-7187(95)00500-5

[32] Del Sarto, N., Isabelle, D.A. and Di Minin, A. (2020) The Role of Accelerators in Firm Survival: An fsQCA Analysis of Italian Startups. Technovation, 90-91, Article ID: 102102. https://doi.org/10.1016/j.technovation.2019.102102

[33] Morse, R.P. (2020) Small Business Survival Capabilities and Policy Effectiveness: Evidence from Oakland. National Bureau of Economic Research, Cambridge.

[34] Clottey, T.A. and Grawe, S.J. (2014) Non-Response Bias Assessment in Logistics Survey Research: Use Fewer Tests? International Journal of Physical Distribution \& Logistics Management, 44, 412-426. https://doi.org/10.1108/IJPDLM-10-2012-0314

[35] Falk, M. (2013) A Survival Analysis of Ski Lift Companies. Tourism Management, 36, 377-390. https://doi.org/10.1016/j.tourman.2012.10.005

[36] Allison, P.D. (2010) Survival Analysis Using SAS ${ }^{\oplus}$ : A Practical Guide. Second Edition. SAS Institute Inc., Cary.

[37] Li, G., Jin, F., Chen, Y., Jiao, J. and Liu, S. (2017) Location Characteristics and Differentiation Mechanism of Logistics Industry Based on Points of Interest: A Case Study of Beijing. Journal of Geographical Sciences, 72, 1091-1103.

[38] Agarwal, R. and Audretsch, D.B. (2001) Does Entry Size Matter? The Impact of the Life Cycle and Technology on Firm Survival. The Journal of Industrial Economics, 49, 21-43. https://doi.org/10.1111/1467-6451.00136

[39] Mario, C., William, G., Roberto, G.G. and Yulia, V.M. (2010) An Introduction to Survival Analysis Using Stata. Third Edition.

[40] Kleinbaum, D.G. and Klein, M. (2012) Survival Analysis: A Self-Learning Text. Third Edition, Springer-Verlag, Berlin.

[41] Cox, D.R. (1972) Regression Models and Life-Tables. Journal of the Royal Statistical Society Series B Statistical Methodology, 34, 187-220. https://doi.org/10.1111/j.2517-6161.1972.tb00899.x

[42] Kiefer, N.M. (1988) Economic Duration Data and Hazard Functions. Journal of Economic Literature, 26, 646-679.

[43] Audretsch, D.B. and Mahmood, T. (1994) The Rate of Hazard Confronting New 
Firms and Plants in U.S. Manufacturing. Review of Industrial Organization, 9, 41-56. https://doi.org/10.1007/BF01024218

[44] Grambsch, P.M. and Therneau, T.M. (1994) Proportional Hazards Tests and Diagnostics Based on Weighted Residuals. Biometrika, 81, 515-526.

https://doi.org/10.1093/biomet/81.3.515

[45] Ono, Y. (2003) Outsourcing Business Services and the Role of Central Administrative Offices. Journal of Urban Economics, 53, 377-395. https://doi.org/10.1016/S0094-1190(03)00006-8

[46] Hull, C.E., Tang, Z., Tang, J. and Yang, J. (2019) Information Diversity and Innovation for Born-Globals. Asia Pacific Journal of Management, 37, 1039-1060. https://doi.org/10.1007/s10490-019-09651-7

[47] Yuan, Q. (2018) Location of Warehouses and Environmental Justice. Journal of Planning Education and Research. https://doi.org/10.1177/0739456X18786392

[48] Hong, J. (2007) Firm-Specific Effects on Location Decisions of Foreign Direct Investment in China's Logistics Industry. Regional Studies, 41, 673-683. https://doi.org/10.1080/00343400601120304

[49] Hong, J. (2007) Transport and the Location of Foreign Logistics Firms: The Chinese Experience. Transportation Research Part A. Policy \& Practice, 41, 597-609. https://doi.org/10.1016/j.tra.2006.11.004

[50] Liang, S., Cao, Y. and Wu, W. (2013) Spatial Pattern Evolution of Port Logistics Enterprises in Shanghai Metropolitan Area. Geographical Research, 32, 1448-1456. 


\section{Appendix}

Table A1. Kaplan-Meier estimates and log-rank tests for survival time.

\begin{tabular}{|c|c|c|c|c|c|}
\hline \multicolumn{3}{|c|}{ The non-parametric test } & \multicolumn{3}{|c|}{ Survival rate } \\
\hline variable & Log-rank & Description & 5 years & 10 years & 15 years \\
\hline \multirow[t]{4}{*}{ Firm type } & 81.66 & $1=\mathrm{TSPs}$ & 0.897 & 0.646 & 0.485 \\
\hline & & $2=\mathrm{WSPs}$ & 0.866 & 0.711 & 0.576 \\
\hline & $(<0.0001)$ & $3=\mathrm{FFCs}$ & 0.819 & 0.593 & 0.487 \\
\hline & & $4=\mathrm{ILSPs}$ & 0.979 & 0.958 & 0.924 \\
\hline \multirow[t]{2}{*}{ Branch } & 10.99 & $1=$ branch & 0.821 & 0.604 & 0.440 \\
\hline & $(0.0009)$ & $0=$ non - branch & 0.882 & 0.655 & 0.536 \\
\hline \multirow[t]{2}{*}{ Ownership } & 7.89 & 1 = foreign-owned & 0.944 & 0.779 & 0.742 \\
\hline & $(0.0050)$ & $0=$ domestic-owned & 0.875 & 0.648 & 0.513 \\
\hline \multirow[t]{2}{*}{ Location } & 43.01 & $1=$ rural area & 0.905 & 0.723 & 0.666 \\
\hline & $(<0.0001)$ & $0=$ urban area & 0.855 & 0.613 & 0.468 \\
\hline \multirow[t]{3}{*}{ Entry timing } & 157.61 & $1=$ early-stage $(1984-2005)$ & 0.932 & 0.809 & 0.664 \\
\hline & & $2=$ medium-stage $(2006-2010)$ & 0.901 & 0.628 & ---- \\
\hline & & $3=$ late-stage (2011-2016) & 0.850 & ---- & ---- \\
\hline \multirow[t]{2}{*}{ Size } & 184.33 & $1=$ large scale & 0.928 & 0.842 & 0.817 \\
\hline & $(<0.0001)$ & $0=$ small scale & 0.857 & 0.581 & 0.410 \\
\hline
\end{tabular}

Note: What in parentheses is p-value, the horizontal line represents that the survival rate is not estimated.

Table A2. Proportional hazard test results.

\begin{tabular}{cccccc}
\hline Variables & P value & Variables & P value & Variables & P value \\
\hline TSPS & 0.2222 & Branch & 0.3965 & $E S$ (Early-stage) & 0.5464 \\
WSPS & 0.5251 & Ownership & 0.5000 & $M S$ (Medium-stage) & 0.2887 \\
FFCs & 0.6447 & Location & 0.2126 & Size & 0.0000 \\
\hline
\end{tabular}

Table A3. Stratified Cox proportional hazard model.

\begin{tabular}{|c|c|c|c|c|c|}
\hline \multirow[b]{2}{*}{ Variables } & \multirow{2}{*}{$\begin{array}{c}(1) \\
\text { Haz. Ratio } \\
\mathrm{e}^{\beta}\end{array}$} & \multirow{2}{*}{$\begin{array}{c}(2) \\
\text { Haz. Ratio } \\
\mathrm{e}^{\beta}\end{array}$} & \multirow{2}{*}{$\begin{array}{c}(3) \\
\text { Haz. Ratio } \\
\mathrm{e}^{\beta}\end{array}$} & \multicolumn{2}{|c|}{ (4) } \\
\hline & & & & $\begin{array}{c}\text { coefficient } \\
\beta\end{array}$ & $\begin{array}{c}\text { Haz. Ratio } \\
\mathrm{e}^{\beta}\end{array}$ \\
\hline \multirow[t]{2}{*}{$T S P S$} & $2.5918^{\star \star}$ & $2.5606^{\star *}$ & $2.5724^{\star \star}$ & $0.8862^{\star \star}$ & $2.4260^{\star *}$ \\
\hline & $(1.0024)$ & $(0.9905)$ & $(0.9963)$ & & $(0.9412)$ \\
\hline \multirow[t]{2}{*}{$W S P_{S}$} & $2.7027^{\star *}$ & $2.6746^{\star *}$ & $2.6850^{* *}$ & $0.9323^{\star *}$ & $2.5404^{\star *}$ \\
\hline & $(1.0741)$ & $(1.0630)$ & $(1.0679)$ & & (1.0118) \\
\hline \multirow[t]{2}{*}{$F F C s$} & $3.4067^{\star * *}$ & $3.3669^{* * *}$ & $3.3811^{* * *}$ & $1.1606^{* * *}$ & $3.1919^{* * *}$ \\
\hline & $(1.3222)$ & $(1.3066)$ & $(1.3134)$ & & $(1.2418)$ \\
\hline Branch & 1.0792 & 1.0783 & 1.0792 & 0.0753 & 1.0783 \\
\hline
\end{tabular}


G. Q. Li et al.

\section{Continued}

\begin{tabular}{cccccc}
\hline & $(0.0908)$ & $(0.0907)$ & $(0.0909)$ & & $(0.0908)$ \\
Ownership & $0.4848^{* *}$ & $0.4869^{* *}$ & $0.4872^{* *}$ & $-0.7090^{* *}$ & $0.4921^{* *}$ \\
& $(0.1497)$ & $(0.1504)$ & $(0.1505)$ & & $(0.1521)$ \\
Location & $0.6530^{* * *}$ & $0.6813^{* * *}$ & $0.6807^{* * *}$ & $-0.3696^{* * *}$ & $0.6910^{* * *}$ \\
& $(0.0408)$ & $(0.0457)$ & $(0.0458)$ & & $(0.0465)$ \\
ES (Early-stage) & $0.2363^{* * *}$ & $0.2380^{* * *}$ & $0.2358^{* * *}$ & $-1.3595^{* * *}$ & $0.2568^{* * *}$ \\
& $(0.0262)$ & $(0.0264)$ & $(0.0276)$ & & $(0.0305)$ \\
MS (Medium-stage) & $0.4998^{* * *}$ & $0.5032^{* * *}$ & $0.5028^{* * *}$ & $-0.5819^{* * *}$ & $0.5588^{* * *}$ \\
& $(0.0365)$ & $(0.0368)$ & $(0.0368)$ & & $(0.0436)$ \\
S_L & & 0.7524 & 0.7553 & $-0.3000^{*}$ & $0.7408^{*}$ \\
S_ES & & $(0.1310)$ & $(0.1320)$ & & $(0.1295)$ \\
Log-likelihood & -8172.0054 & -8170.6399 & -8170.6085 & & -8162.7875 \\
S_MS & 6785 & 6785 & 6785 & & 6.5785 \\
\hline
\end{tabular}

Note: Standard error in parentheses ${ }^{* * *} \mathrm{p}<0.01,{ }^{* *} \mathrm{p}<0.05,{ }^{*} \mathrm{p}<0.1$ exact marginal likelihood Stratified by Size. In the sample, the survival time of six LSPs is 0 , which were excluded. 